\title{
Genetic-guided pharmacotherapy for venous thromboembolism: a systematic and critical review of economic evaluations
}

\author{
Ka Keat Lim $\mathbb{D}^{1,2} \cdot$ Rositsa Koleva-Kolarova ${ }^{3} \cdot$ Philip Chowienczyk ${ }^{4} \cdot$ Charles D. A. Wolfe $\mathbb{1}^{1,2,5} \cdot$ Julia Fox-Rushby $\mathbb{(}^{1,2}$
}

Received: 19 January 2021 / Revised: 12 May 2021 / Accepted: 19 May 2021 / Published online: 15 June 2021

(c) The Author(s) 2021. This article is published with open access

\begin{abstract}
Despite the known contributions of genes, genetic-guided pharmacotherapy has not been routinely implemented for venous thromboembolism (VTE). To examine evidence on cost-effectiveness of genetic-guided pharmacotherapy for VTE, we searched six databases, websites of four HTA agencies and citations, with independent double-reviewers in screening, data extraction, and quality rating. The ten eligible studies, all model-based, examined heterogeneous interventions and comparators. Findings varied widely; testing was cost-saving in two base-cases, cost-effective in four, not cost-effective in three, dominated in one. Of 22 model variables that changed decisions about cost-effectiveness, effectiveness/relative effectiveness of the intervention was the most frequent, albeit of poor quality. Studies consistently lacked details on the provision of interventions and comparators as well as on model development and validation. Besides improving the reporting of interventions, comparators, and methodological details, future economic evaluations should examine strategies recommended in guidelines and testing key model variables for decision uncertainty, to advise clinical implementations.
\end{abstract}

These authors contributed equally: Ka Keat Lim, Rositsa KolevaKolarova

Supplementary information The online version contains supplementary material available at https://doi.org/10.1038/s41397021-00243-7.

Julia Fox-Rushby

julia.fox-rushby@kcl.ac.uk

1 School of Population Health and Environmental Sciences, Faculty of Life Sciences and Medicine, King's College London, London, UK

2 National Institute for Health Research (NIHR) Biomedical Research Centre, Guy's and St Thomas' NHS Foundation Trust and King's College London, London, UK

3 Health Economics Research Centre, Nuffield Department of Population Health, University of Oxford, Oxford, UK

4 Cardiovascular Division, Department of Clinical Pharmacology, King's College London School of Medicine, St Thomas' Hospital, London, UK

5 National Institute for Health Research (NIHR) Collaboration for Leadership in Applied Health Research and Care (ARC) South London, London, UK

\section{Introduction}

Venous thromboembolism (VTE), comprising deep vein thrombosis (DVT) and pulmonary embolism (PE), is a major cardiovascular disease (CVD) [1]. Patients with previous VTE are at high risk of recurrence [1]. Factor V Leiden (FVL) and prothrombin G20210A (PT-G20210A) are genetic risk factors associated with twice the risk of recurrence in carriers compared to non-carriers [2]. To reduce the risk of recurrence, patients are often treated with anticoagulants $[2,3]$ which may cause bleeding as a sideeffect, especially among carriers of gene variants e.g. CYP2C9 $2 * / 3 *$ and Vitamin K epoxide reductase complex 1 associated with twice the risk of bleeding compared to non-carriers [4].

Despite the known contribution of genes, guiding anticoagulation therapy based on genetic testing-prolonged anticoagulation for carriers of genes associated with higher risk of recurrence and genotype-guided dosing for carriers of genes associated with higher risk of bleedinghas not been routinely implemented in VTE management. This may be due to clinical guidelines that recommend not to test for genes associated with higher risk of recurrent VTE $[2,3,5]$, especially among patients with transient risk related to oral contraception, major surgeries and chemotherapy. Genetic testing is currently recommended only 
for patients with persistent risk factors such as those related to age or lifestyle, who have first-degree relatives with VTE $[2,3]$, and the decision to initiate or to stop anticoagulation does not rely on the test finding alone [2].

Nevertheless, with the discovery of more genes associated with recurrent VTE $[6,7]$ and the increasing accessibility of genetic test to clinicians and patients [8], whether it is cost-effective to incorporate these tests in clinical settings should be examined and discussed. The extent and the quality of the existing economic evidence can be ascertained via a systematic review.

The most recent review of economic evaluations of genetic-guided pharmacotherapy for VTE, published in 2012 [9] found seven studies based on models, but had several gaps. Firstly, only four studies identified focused on patients with persistent risk factors, for whom selective testing based on family history is currently recommended $[2,3]$. These modelling studies rarely involved clinicians in the model development and hence likely did not reflect actual clinical contexts. In addition, the review did not elaborate the clinical contexts being considered and the consequences of genetic testing accounted for, to allow clinicians to assess whether the findings would be relevant for their settings. The review did not examine variables that may affect the cost-effectiveness of genetic testing to inform the development of future models. Finally, the review appraised the reporting but not the methodological quality.

To address these gaps, our study identifies and analyses economic evaluations of pharmacogenetic-guided pharmacotherapy in patients with VTE to present (1) details on the provision of interventions and comparators to reflect the clinical contexts, (2) which consequences of genetic testing were accounted for, (3) the model variables that may influence cost-effectiveness and (4) the gaps in both reporting and methodological qualities. By addressing these gaps, clinicians and policy makers interested in implementing pharmacogenetic-guided pharmacotherapy in patients with VTE would be better informed of the strengths and weaknesses of existing economic evidence and can identify future research and policy measures to support the implementation.

\section{Methods}

This study is part of a larger systematic review aiming to assess the economic evidence of genetic-guided pharmacotherapy in patients with CVD (prospectively registered on PROSPERO ID: CRD42019144579 [10]).

\section{Search strategies and study selection}

The methodological details are available on the register and in Appendix 1, guided by the Preferred Reporting Items for Systematic Reviews and Meta-Analyses statement (Appendix 2) [11]. Briefly, we systematically searched three general bibliographic databases (Medline, Embase, Web of Science Core Collection) and three subject-specific bibliographic databases (Econlit, NHS Economic Evaluation Database, Health Technology Assessment) from inception until 29 June 2020 (Appendix 3). The database searches were supplemented [12] by searching the websites of four health technology assessment (HTA) agencies (UK NICE; Canadian CADTH; French HAS; Dutch ZonMw). We also searched reference lists of included articles and systematic or narrative review articles, and citations of included articles on Scopus. The titles and abstracts were double screened for potential eligibility after duplicates were removed, with disagreements resolved through discussion. Articles were included if they: reported a full economic evaluation based on models, trials or quasi-observational studies; and focused on genetic testing followed by pharmacotherapy for patients with VTE, where the VTE was not due to transient risk factors. Articles were excluded if they considered hypothetical genetic tests, used animals, or were review articles, study protocols, editorials, commentaries, opinions, conference abstracts or letters.

\section{Data extraction}

We extracted author details, study design, sample characteristics, details on provision of genetic testing and comparator interventions, costs, outcome measures, analyses performed, the model variables and the base-case conclusion on cost-effectiveness based on the local willingness-to-pay (WTP) threshold. Base-case is the scenario which operationalises the best available estimates of the model variables as identified by the authors of the studies.

The two sections on details on the provision of genetic testing and its comparators were operationalized from the Template for Intervention Description and Replication [13] (Appendix 4).

In extracting the model variables, we indicated whether the variables were tested in one-way deterministic sensitivity analyses (DSA) and which, within the range tested, were influential in changing the base-case conclusion. Oneway DSA is a simple sensitivity analysis in economic evaluations where a point estimate of a model variable is varied while keeping the others constant, to examine whether the variable could change the base-case conclusion (e.g. from being cost-effective to not cost-effective). 
To assess the range of consequences of genetic testing captured by the studies, the impact inventory recommended by the Second Panel on Cost-effectiveness in Health and Medicine was used $[14,15]$. The impact inventory is a list of 21 consequences an intervention may have inside and outside of healthcare sector $[14,15]$.

\section{Quality assessment}

Reporting quality was assessed using the checklist developed by the Second Panel (Appendix 5, with guidelines quoted from [14, 15] to support judgement). Each of the 47 items was rated "Yes", "No", "Partial" or "Not Applicable".

Methodological quality was assessed using the extended version of Consensus Health Economic Criteria List (CHEC-Extended) [16, 17]. Each of the 20 items, as recommended [16, 17] was rated "Yes/rather yes", "No/ rather no" or "Unclear".

Double data extraction and double quality ratings were undertaken independently, with reference to a third reviewer on the interpretation of items for the first two papers and when any discrepancies could not be resolved for the remaining eight papers.

\section{Data analyses and presentation}

To provide an overview, the study design and sample characteristics are presented as counts and percentages. Details of the interventions and comparators are visualised in a network diagram. The consequences of genetic testing accounted for in the studies are presented by study perspective, as recommended [14, 15].

A narrative synthesis of the economic evaluation findings are provided in a permutation matrix [18]. This $3 \times 3$ matrix presents each intervention in terms of whether its relative costs and relative effects are better, no different or worse from the comparator in the base-case. Interventions that appear in the bottom left of the matrix are less costly and more effective than the comparators and hence would be favoured for adoption in clinical practice. In contrast, interventions that appear in the top right are more costly and less effective than the comparators and hence would be rejected. Interventions that appear elsewhere in the matrix would require trading off costs and benefits, and comparison with a WTP threshold value prior to an adoption decision.

To examine the model variables influential in changing the base-case conclusion of the economic evaluations, we first organised the model variables into four categories: effectiveness, epidemiology, cost and utility. The epidemiological and the cost variables were subcategorised according to the framework of economic evaluations of genetic testing [19] and the cost categories in a related systematic review [20], respectively. Based on these categories and subcategories, we presented the number of model variables reported, tested in one-way DSA, with findings reported and were influential.

To examine the reporting and methodological quality of the included studies, we presented the percentage of items with each rating.

\section{Results}

\section{Study inclusion}

Of 5853 articles identified, 4733 were unique. From the unique articles, 4333 were removed after title and abstract screens and a further 392 after full-text screens. The top three reasons for exclusion from full-text screens were: not empirical study (e.g. editorials), not economic evaluation and economic evaluations on CVDs other than VTE. Citation searches of included papers identified two additional papers, resulting in a total of ten papers (Fig. 1).

\section{Study characteristics}

Figure 2 summarises characteristics of studies (see Appendix 6 for details). Most studies were published between 2009 and 2015, and were equally distributed between North America [21-25] and Europe [26-30].

All studies used models to assess the costs and the effects of genetic-guided pharmacotherapy. Half declared adopting a societal perspective; four [21, 25-27] assessed costs and effects over a lifetime and one [22] over 2 years. Only one study [29] reported its model development process and face validation with clinical experts.

Seven studies performed a cost-utility analyses (CUA), two a cost-effectiveness analyses (CEA) and one both a CUA and CEA. Most studies [21, 25-27, 29, 30] simulated patients aged 20-60 years at baseline; one study [22] simulated paediatric patients (2-18 years old); one study [30] simulated multiple patient subgroups aged 30-70 years. Contrary to the clear reporting of age, most studies did not explicitly report the gender of their simulated patients. No study reported the family history or CVD risk factors of their simulated patients.

\section{Interventions and comparators}

The interventions and comparators were heterogeneous (Fig. 3). Interventions differed in the type of genes tested, the number of genes tested and, among eight studies that tested multiple genes (with or without anticoagulant biomarkers, e.g. activated protein $\mathrm{C}$ ), the test sequence. On the types of genes, seven studies [21, 22, 25-27, 29, 30] 


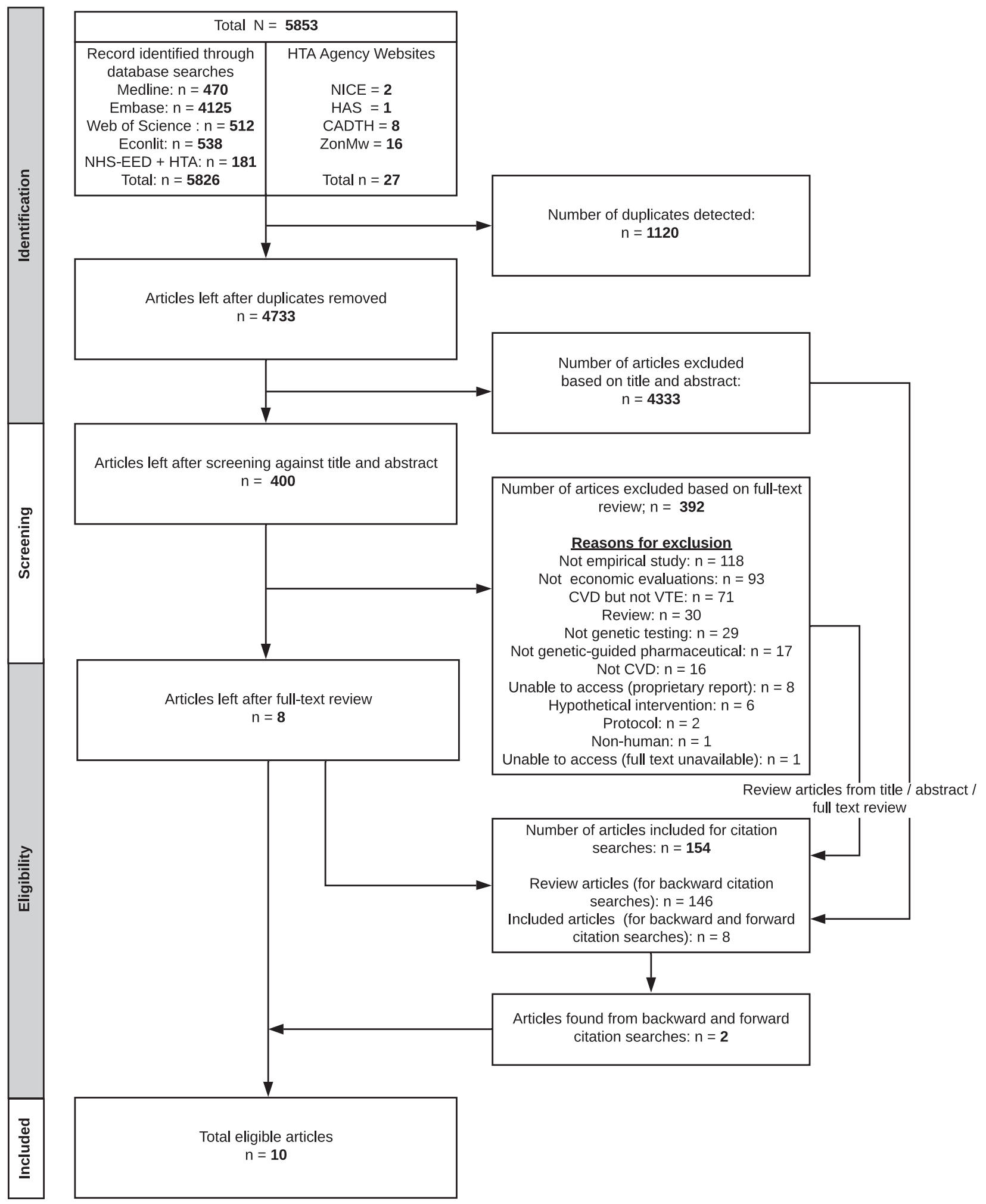

Fig. 1 PRISMA flow chart. The flow chart indicates the flow of study selection, from searching the bibliographic databases and the websites of health technology assessment (HTA) agencies, to study screening and the inclusion of 10 eligible articles.

examined testing of genes associated with higher risk of recurrent VTE, whereas three $[23,24,28]$ examined testing of genes associated with higher risk of bleeding with anticoagulation. Patients tested positive with the former were treated with prolonged anticoagulation therapy ( $\geq 6$ months) whereas patients tested positive with the latter 


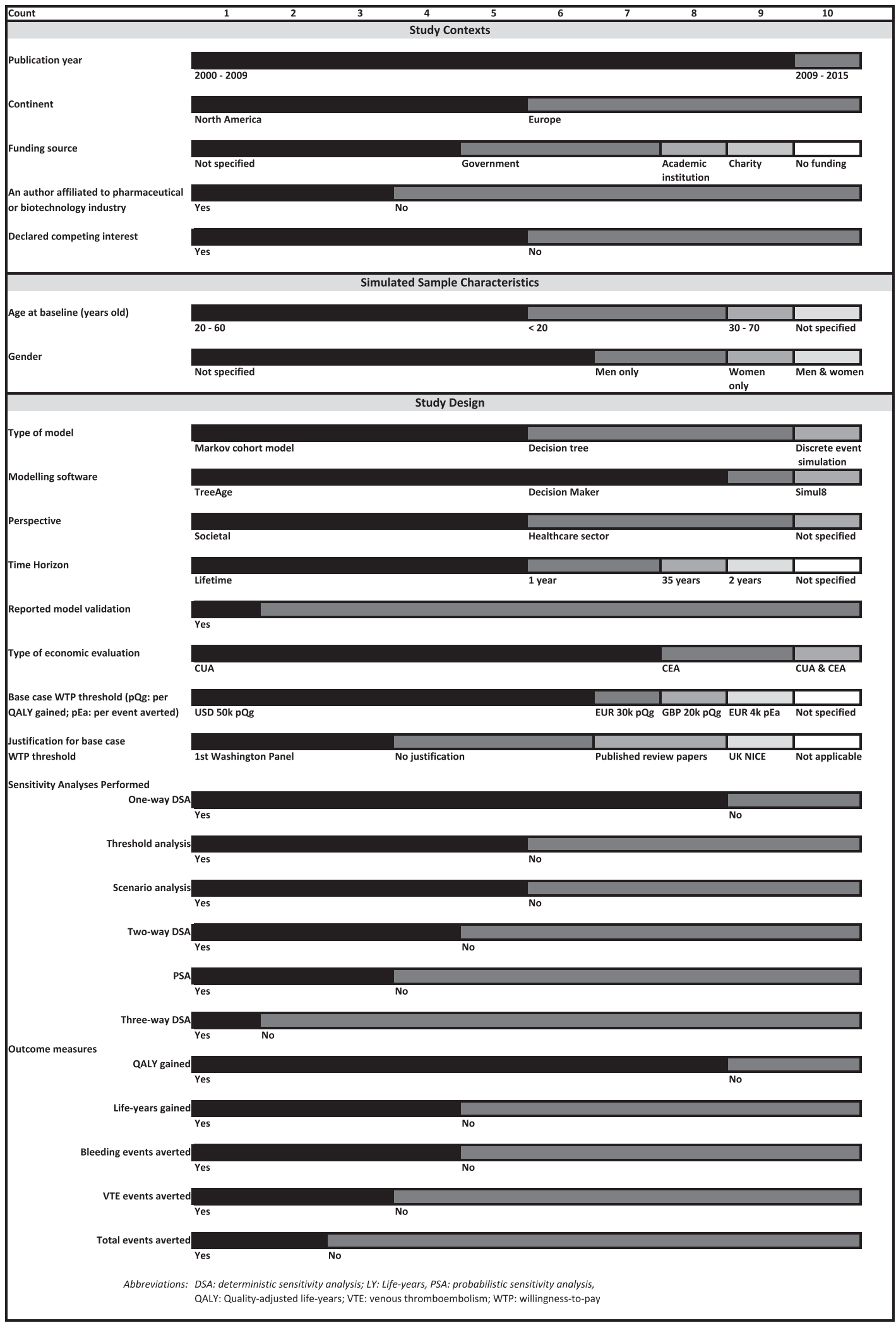

Fig. 2 Characteristics of included studies. The horizontal bar charts represent the number of studies with each characteristic (study context, simulated sample characteristic and study design). 


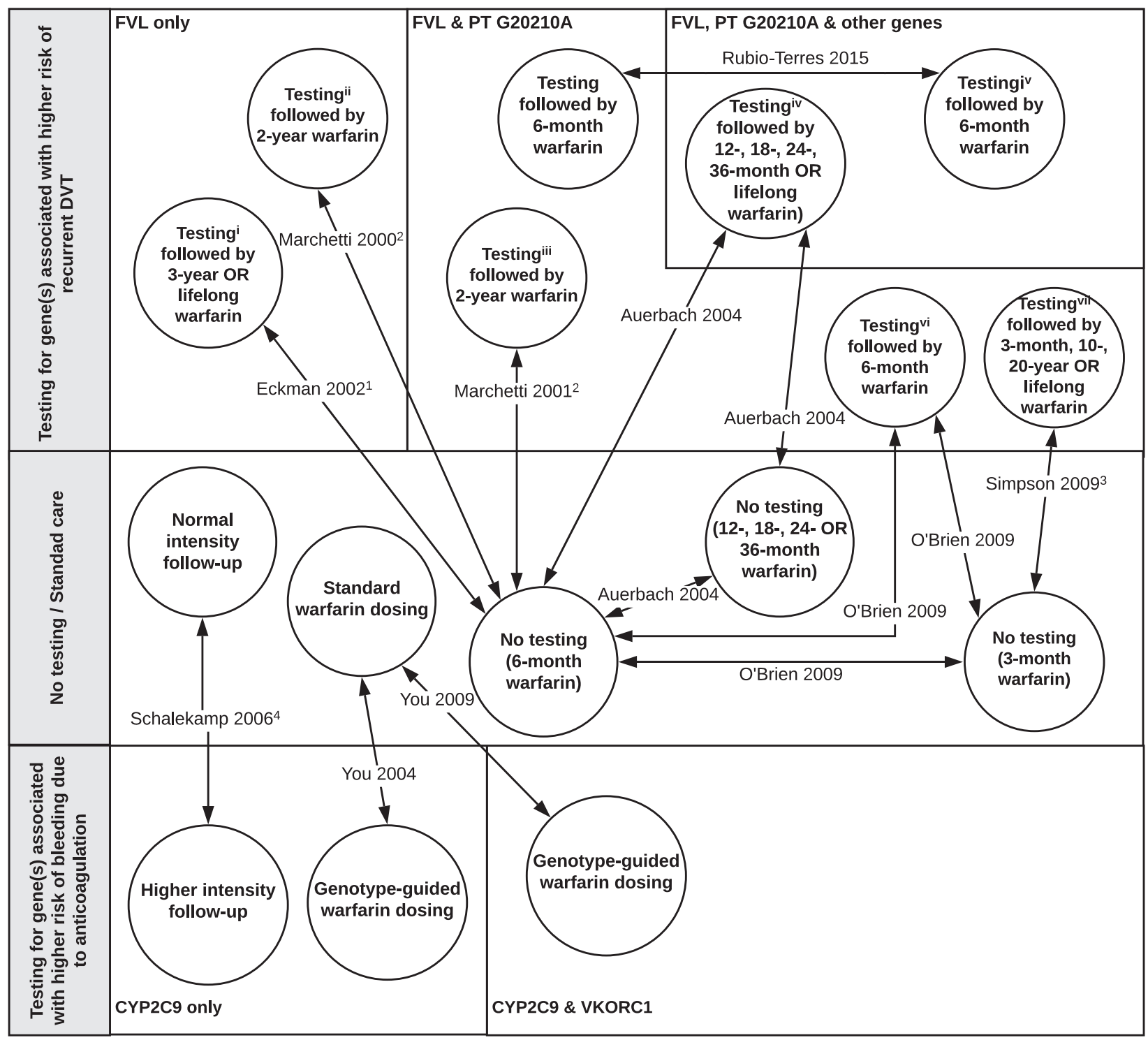

FVL: Factor V Leiden; PT: Prothrombin G20210A
i. Patients tested for FVL only if they are first tested to be resistant to Activated Protein C (APC)
ii. Patients tested using second-generation APC-resistance test and for FVL
iii. Patient tested for PT only if they are first tested positive for FVL
iv. Patients tested for mutations in 3 genes (FVL, PT and dihydrofolate reductase), followed by anticardiolipin antibody, antithrombin III, and protein $C$ and $S$ levels after initial anticoagulation.
v. Patients tested for 12 variants located on 7 genes - FVL, PT, FXII, FXIII, ABO, Serpin A10 and Serpin C1
vi. Patients tested for FVL, PT, protein C, protein $S$, and antithrombin activity levels
vii. Patients tested for FVL, PT, lupus anticoagulant, anticardiolipin antibody, antithrombin decifiency, protein C deficiency or protein S deficiency or lupus-like anticoagulants

\begin{abstract}
All studies did not specify the sex of their simulated samples, except (1) Eckman et al 2002 simulated women only; (2) Marchetti et al 2000 and Marchetti et al 2001 simulated men only; (3) Simpson et al 2009 simulated men and women separately.
\end{abstract}

All studies used warfarin as the anticoagulant, except (4) Schalekamp et al 2006 which used acenocoumarol.

Fig. 3 Overview of interventions and comparators. The network diagram summarises the types of genetic-guided pharmacotherapy and the comparators for the 10 included studies.

had genotype-guided dosing [23, 24] or higher intensity of follow-up [28]. In offering the test or deciding the duration of anticoagulation, no consideration of family history or risk factors other than the test finding was mentioned. On the number of genes tested, earlier studies [21, 24, 26, 28] tested only a single gene, later studies tested two $[22,23,27,30]$ or three [25] genes and the latest study [29] tested seven genes. On the test sequence, four studies 
offered sequential testing-anticoagulant biomarker before gene testing [21, 26], gene testing before anticoagulant biomarker [25] or one gene after another [27]; four studies offered simultaneous testing of multiple genes [23, 29] or genes with biomarkers [22, 30].

The comparators differed with regards to the interventions examined. Among studies that tested genes associated with higher risk of recurrent VTE, the comparators were mostly no-testing, with duration of anticoagulation (3 months to 3 years) shorter than that for patients tested positive. Only in one study [29], instead of no-testing, the comparator was testing fewer genes; this was the only study where the duration of anticoagulants did not differ between intervention and comparator. Meanwhile, for studies that tested genes associated with higher risk of bleeding, their comparators were standard warfarin dosing [23, 24] or normal intensity of follow-up [28].

All included studies used warfarin as the anticoagulant except one [28] that used acenocoumarol. None of the studies explicitly reported the setting in which the testing took placetwo [24, 28] mentioned "anticoagulation clinics" without specifying whether these clinics were located in primary or secondary care settings; one study [30] mentioned testing was available in specialist laboratories or hospitals, but did not state which case was modelled. No study reported the testing procedures (e.g. blood, saliva) or the providers involved (e.g. nurse, lab technician). All studies assumed $100 \%$ uptake of genetic testing and only two studies [26, 29] considered less than perfect adherence to anticoagulation.

\section{Consequences of genetic testing accounted for}

Based on the impact inventory (Appendix 7), all studies accounted for $>1$ consequence of genetic testing within the formal healthcare sector. The three most common consequences were health-related quality-of-life $(n=8)$, future-related medical costs $(n=6)$ and other health effects $(n=5)$. No study accounted for costs incurred by third-party payers, patients out-of-pocket spending or future unrelated medical costs. Of five studies that declared societal perspective, only three accounted for consequences outside formal healthcare sector, specifically labour marketing earnings loss [22, 26, 27] and transportation costs for physician visits and anticoagulation monitoring [22].

\section{Economic evaluation findings}

The base-case conclusions were distributed across three corners of the matrix (Fig. 4). The robustness of some basecase conclusions was further examined in different scenarios (scenario analyses) or in repeated simulations with varied model variables to estimate the probability of the intervention being cost-effective (probabilistic sensitivity analyses (PSA)).

Of the seven studies testing for genes associated with higher risk of recurrent VTE, six were at the bottom left or the top left of the matrix, concluding that at base-case, testing may be cost-saving, cost-effective or not costeffective. Among the two that concluded cost-saving, one [21] showed via scenario analyses that genetic testing compared to no-testing remained cost-saving even with different assumptions on the persistence of recurrent VTE risk; while the other [29] demonstrated in PSA that testing for seven genes had a $100 \%$ probability of cost-saving compared to testing for two genes. One study [30] simulated multiple patient subgroups and found that in patients with PE, testing was cost-effective regardless of age and gender whereas in patients with DVT, testing was costeffective in men $<70$ and women $<50$ years old. In the PSA of this study, however, testing was cost-effective only in $30-60 \%$ simulations among the subgroups. Three other studies also found testing cost-effective, with similar incremental cost-effectiveness ratios (USD11,100-13,624 per QALY gained [25-27]) over a patient's lifetime despite testing different number of genes. None, however, examined the robustness of their base-case conclusion in scenario analyses or PSA. Meanwhile, at the top right corner, testing was dominated by no-testing in the only study that simulated paediatric patients [22].

All three studies that analysed testing for genes associated with higher risk of bleeding were in the top left corner. One [23] concluded that testing was not costeffective and found, using PSA, a low probability of testing being cost-effective $(19.1 \%)$ or cost-saving $(18.7 \%)$. The other study had two base-cases-testing was cost-effective in the base-case with higher prevalence but not costeffective in the base-case with lower prevalence of high-risk gene variants. However, it did not further examine the robustness of its base-case conclusions in PSA. The last study [24], without a WTP threshold, did not conclude on cost-effectiveness.

\section{Influential model variables}

A total of 309 model variables were reported, approximately half were epidemiological variables, followed by cost, utility and effectiveness or relative effectiveness of interventions.

Overall, 53\% variables (164/309) were tested in one-way DSA. Of 134 variables with findings reported, 22 were influential in changing the base-case conclusion, with the most frequent $(5 / 22)$ being effectiveness or relative effectiveness of the intervention. However, these effectiveness estimates were not based on any trials of genetic testing in patients with VTE. Instead, they were based on randomised trials of genetic testing 
Fig. 4 Findings of economic evaluations. The $3 \times 3$ matrix presents each intervention in terms of whether its relative costs and relative effects are higher/better $(+)$, no different (0) or lower/worse (-) from the comparator in the base-case.

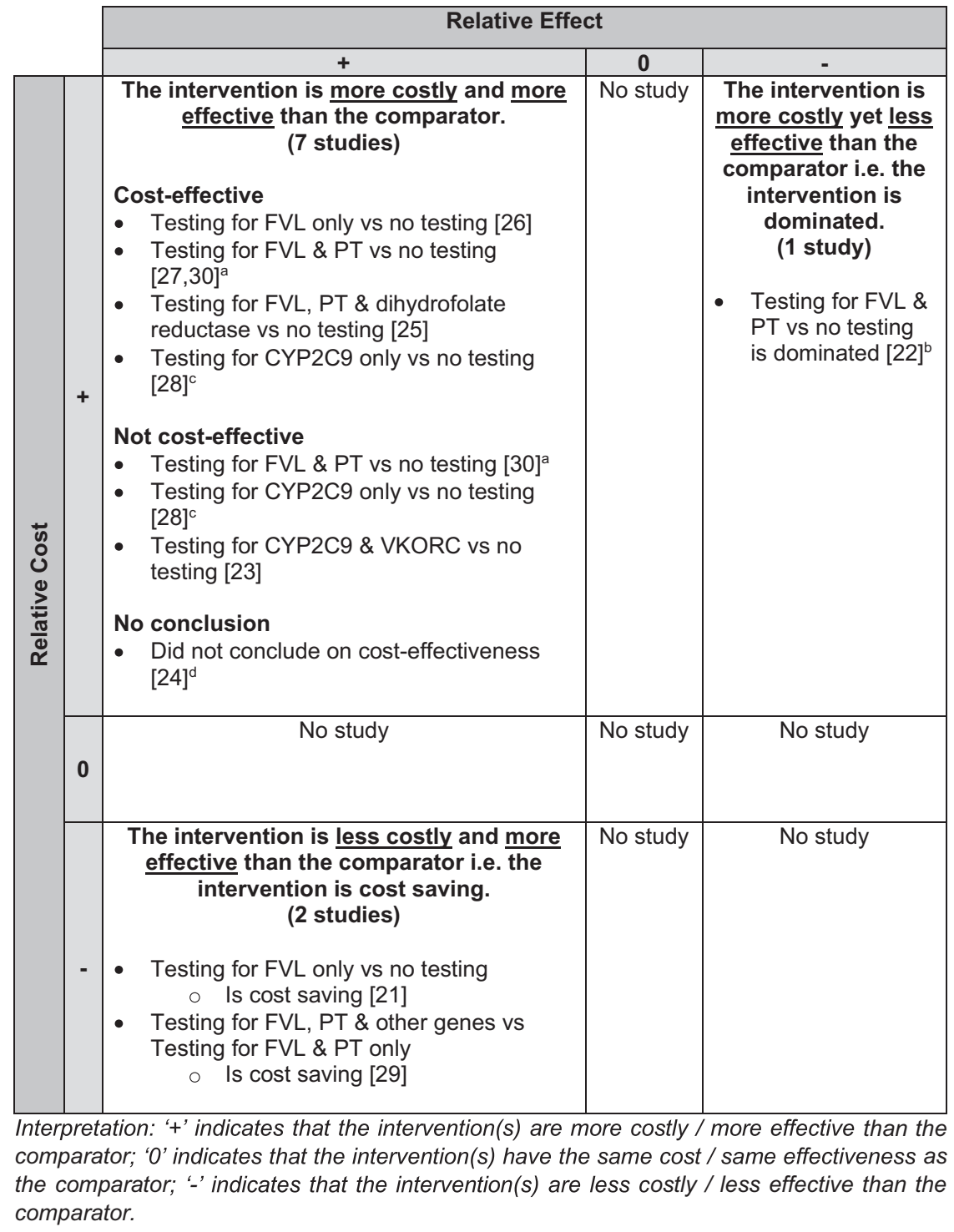

All conclusions on cost-effectiveness are for the base case, according to the willingness-topay (WTP) thresholds of the individual studies. in non-VTE patients (23); observational studies and/or a randomised trial of prolonged warfarin in preventing recurrent VTE [21, 26, 27]; or assumption without elaboration, for the reduction in out-of-range INR [28]. The other influential variables were the risk of bleeding or recurrent VTE (with or without high-risk gene variants), prevalence of high-risk gene variants, cost of genetic testing, cost of warfarin monitoring, cost of treatment of bleeding and utility while taking warfarin (Table 1).

\section{Reporting quality}

Overall, 34-66\% of the 47 items in Second Panel's reporting checklist were rated "Yes" (Fig. 5a and Appendix 8). Ten items were reported by all studies (e.g. type of analysis, software used) whereas six were not reported by any study (e.g. intervention details, results of model validation). Other items less commonly reported were methods for obtaining data, critique of data quality and discussion of ethical implications of genetic testing.

\section{Methodological quality}

Overall, 55-85\% of the 20 items in CHEC-Extended were rated "Yes/rather yes" (Fig. 5b and Appendix 9). Six items were reported by all studies (e.g. economic study design) whereas one item (i.e. ethical and distributional issues) was not discussed appropriately in any study. Other items less reported were structural assumptions, validation methods and discussion of generalisability. 
Table 1 Model variables reported, tested and shown to change base-case conclusion on cost-effectiveness.

\begin{tabular}{|c|c|c|c|c|}
\hline \multirow{2}{*}{$\begin{array}{l}\text { Type of model input } \\
\text { Variables }\end{array}$} & \multicolumn{4}{|c|}{ Number of model variables } \\
\hline & Reported & $\begin{array}{l}\text { Tested in one- } \\
\text { way DSA }\end{array}$ & $\begin{array}{l}\text { Findings } \\
\text { available }\end{array}$ & $\begin{array}{l}\text { Changed } \\
\text { conclusion }\end{array}$ \\
\hline $\begin{array}{l}\text { Effectiveness/relative effectiveness of } \\
\text { intervention }\end{array}$ & 13 & 10 & 7 & 5 \\
\hline \multicolumn{5}{|l|}{ Epidemiological Variables } \\
\hline Assay characteristics & 36 & 34 & 34 & 0 \\
\hline Prevalence of high-risk gene variant(s) & 11 & 5 & 4 & 2 \\
\hline Prevalence of biomarkers ${ }^{\mathrm{a}}$ & 3 & 0 & 0 & 0 \\
\hline \multicolumn{5}{|c|}{ Baseline/relative risk in those with high-risk gene variant(s) } \\
\hline Recurrent $\mathrm{VTE}^{\mathrm{b}}$ & 8 & 5 & 5 & 1 \\
\hline Bleeding $^{\mathrm{c}}$ & 3 & 2 & 1 & 1 \\
\hline \multicolumn{5}{|c|}{ Baseline/relative risk in those without high-risk gene variant(s) } \\
\hline Recurrent VTE ${ }^{\mathrm{a}}$ & 56 & 25 & 22 & 3 \\
\hline Bleeding $^{\mathrm{c}}$ & 42 & 21 & 14 & 4 \\
\hline VTE complications $^{\mathrm{d}}$ & 12 & 1 & 1 & 0 \\
\hline Other epidemiological variables ${ }^{\mathrm{e}}$ & 6 & 6 & 4 & 1 \\
\hline \multicolumn{5}{|l|}{ Cost/resource use/unit price } \\
\hline Genetic testing & 11 & 8 & 7 & 2 \\
\hline Testing of biomarkers ${ }^{f}$ & 1 & 0 & 0 & 0 \\
\hline Anticoagulation monitoring ${ }^{\mathrm{g}}$ & 16 & 7 & 4 & 1 \\
\hline Treatment of VTE & 23 & 12 & 10 & 0 \\
\hline Treatment of VTE complications ${ }^{\mathrm{d}}$ & 12 & 0 & 0 & 0 \\
\hline Treatment of bleeding & 23 & 11 & 8 & 1 \\
\hline Death & 1 & 1 & 1 & 0 \\
\hline \multicolumn{5}{|l|}{ Utility } \\
\hline Anticoagulation & 6 & 4 & 3 & 1 \\
\hline VTE & 7 & 3 & 2 & 0 \\
\hline VTE complications ${ }^{\mathrm{d}}$ & 6 & 1 & 1 & 0 \\
\hline Bleeding & 8 & 5 & 3 & 0 \\
\hline No event/off-treatment & 2 & 2 & 2 & 0 \\
\hline Other utility $^{\text {h }}$ & 3 & 1 & 1 & 0 \\
\hline Total variables & 309 & 164 & 134 & 22 \\
\hline \multicolumn{5}{|c|}{$\begin{array}{l}\text { aPrevalence of lupus anticoagulant, anticardiolipin antibody and antithrombin, protein } \mathrm{C} \text { or protein } \mathrm{S} \\
\text { deficiency. }\end{array}$} \\
\hline \multicolumn{5}{|c|}{${ }^{\text {b}}$ Venous thromboembolism and deaths due to venous thromboembolism. } \\
\hline \multicolumn{5}{|c|}{${ }^{\mathrm{c} H a e m o r r h a g e}$ and deaths due to haemorrhage. } \\
\hline \multicolumn{5}{|c|}{${ }^{\mathrm{d}}$ Includes non-haemorrhagic stroke and post-thrombophlebitis syndrome. } \\
\hline \multicolumn{5}{|c|}{${ }^{\mathrm{e}}$ Compliance to anticoagulation and probability of deaths. } \\
\hline \multicolumn{5}{|c|}{$\begin{array}{l}\text { f Testing for Activated Protein C (in Eckman et al. } 2002 \text { [21], a patient must first be shown to be sensitive to } \\
\text { Activated Protein C to be eligible for genetic testing). }\end{array}$} \\
\hline \multicolumn{5}{|c|}{${ }^{\mathrm{g}}$ Cost of anticoagulation medication and/or monitoring. } \\
\hline
\end{tabular}

\section{Discussion}

Our review systematically examined economic evaluations of genetic-guided pharmacotherapy in patients with VTE driven by persistent risk factors. We found ten studies, six more than the previous review [9]. These studies, modelling a heterogeneous network of interventions and comparators, examined genetic testing followed by prolonged anticoagulation (for genes associated with higher risk of recurrent VTE) or genetic testing followed by genotype-guided dosing or higher follow-up intensity (for genes associated with higher risk of bleeding). All studies were models, 
Fig. 5 The vertical bar charts summarise the ratings for reporting and methodological quality respectively. The y-axes of both charts represent the percentage of items whereas the numbers within the bars indicate the number of items with each rating. a Reporting and b methodological quality ratings. (a) Reporting quality based on Washington Panel's Checklist (47 items)

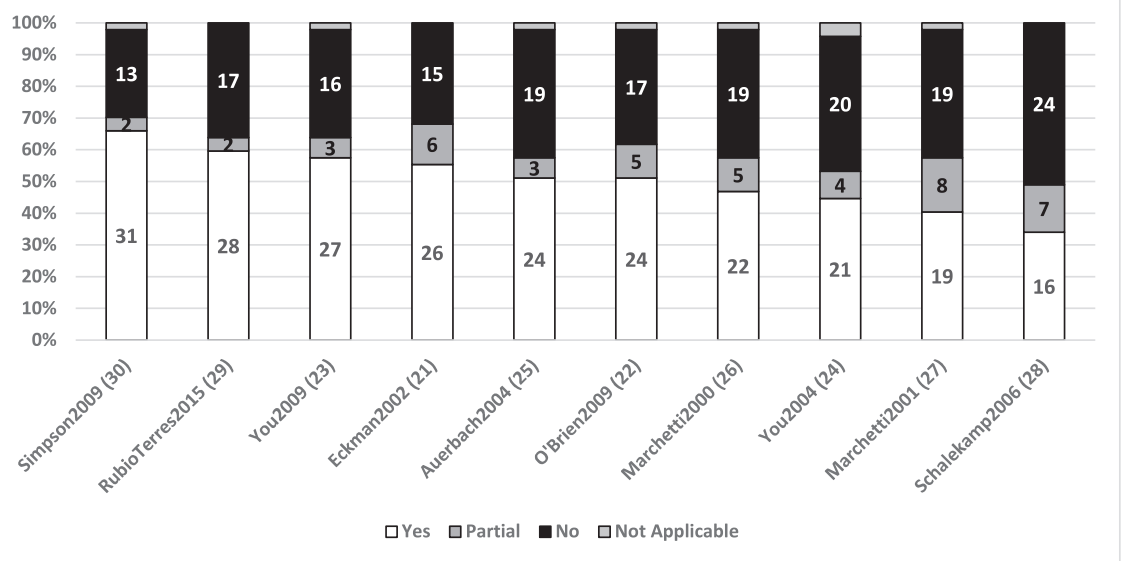

(b) Methodological quality based on CHEC-Extended (20 items)

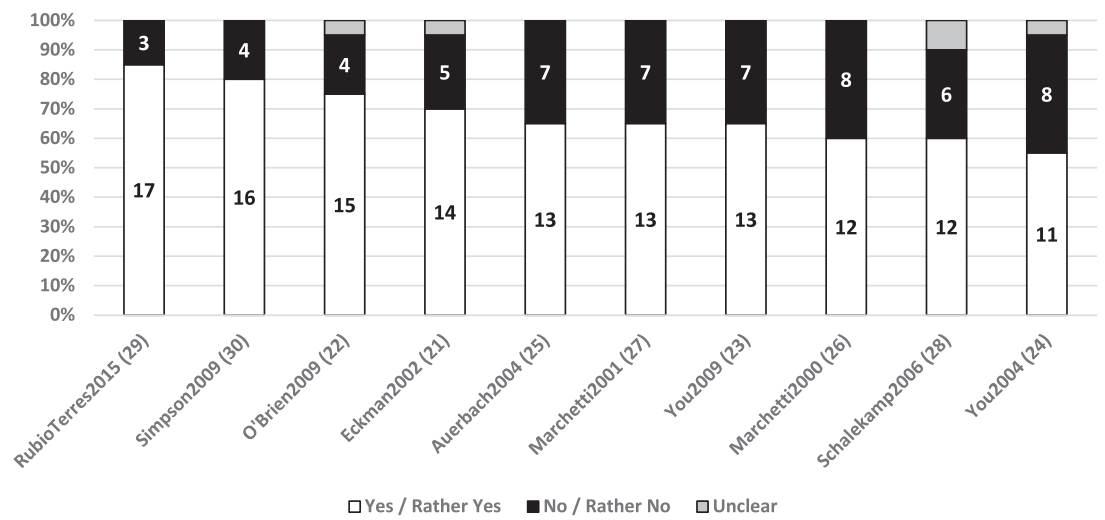

The number within each bar indicates the number of items with each rating, in descending order of Yes (a) or "Yes / Rather Yes" (b). mostly accounted for consequences of genetic testing within formal healthcare sector. Gaps in reporting and methodological qualities were apparent, with studies lacking details on the delivery of interventions and comparators as well as on model development and validation. Results on costeffectiveness were mixed, ranging across being cost-saving, cost-effective, to being too costly for existing costeffectiveness thresholds and dominated by comparators. The heterogeneity of interventions and mixed findings means it is not yet clear which type of genetic testing is cost-effective or cost-saving.

As the studies were published before recent clinical guidelines [2, 3], it is not surprising that none modelled the currently recommended test strategies [2]—-to offer testing only to those with strong family history and to decide on the initiation or the continuation of anticoagulation therapy on the basis of other risk factors as well as the test finding. None examined testing for extended list of genes recently found to be associated with higher risk of recurrent VTE for which a multiple-gene panel is available [6, 7]. In addition, none explored testing both sets of genes, to reduce the risk of recurrent VTE as well as bleeding. These are potential test strategies that future economic evaluations could examine.

Of the list of 22 model variables found to change findings on cost-effectiveness, the most frequent variable was effectiveness or relative effectiveness of the intervention. The quality of data used for effectiveness was not high, however, as they lack directly relevant trial data in patients with VTE. Existing trials [31] and meta-analysis of trials [32, 33], although some do not demonstrate benefits of genetic-guided pharmacotherapy of anticoagulant, are not specific to patients with VTE. While some may consider that the incremental risk attributable to currently known genes (FVL and PT-G20210A) are not high enough to change treatment decision [8], this may need to be revisited when a new gene or a panel of genes associated with risk higher than currently known ones are discovered. New technologies suggest this is imminent [6, 7]. In this case, our list of variables could inform the design of data collection forms for trials as well as the cost-effectiveness models. 
The list of variables has two shortcomings, nevertheless. First, it is likely incomplete. For example, three studies $[22,26,27]$ accounted for loss of earnings due to VTE but did not report the values. Secondly, details on costs were lacking. For example, only one study specified whether the cost of genetic tests included consumables or overheads [24], with the rest ambiguous; poor reporting of the setting in which the genetic testing was delivered made it challenging to account for the variability in costs between different delivery settings. Finally, the uptake of genetic testing was notably absent since all studies assumed perfect uptake. This is unlikely to match reality, especially if positive results cause stress and anxiety [34]. Future evaluations should account for test uptake.

The impact inventory indicates that some relevant consequences related to genetic testing or VTE were not accounted for (e.g. patient time). Accounting for these consequences may make testing more cost-effective if pharmacotherapy following testing can prevent or reduce them. One consequence of genetic testing not listed in the impact inventory is the psychological impact of knowing the genetic test findings (the "value of knowing" [35]). The value of knowing may also extend to family members (of those tested positive with genes associated with higher risk of recurrent VTE) who receive cascade testing, although the clinical benefit of cascade testing for family members is still unclear [2, 3]. Neither the value of knowing nor cascade testing was examined by the included studies, hence how these may have changed the findings is also unknown and can be explored in future studies.

Our review has several limitations. First, it excluded patients with transient risk factors of VTE, e.g. those taking contraceptives or undergoing major surgeries. However, excluding them allow us to provide a focused summary of evidence for those with persistent risk factors. Second, our findings may not be generalisable to settings where direct oral anticoagulants is used [36, 37], as no included studies used them as the anticoagulant. Third, as all studies examined VTE as a whole, it remains unknown whether there is any VTE subtype for which genetic-guided pharmacotherapy may be more favourable. Next, in the absence of recommended rating options, we used our own operationalization of the Second Panel's reporting checklist based on the Panel's publications [14, 15]. We shared the guidance we compiled to support our judgement in Appendix 5. Fourth, while we differentiated reporting and methodological quality by using two different checklists, both checklists ultimately relied on reporting. This may explain why included studies with better reporting quality also appeared to have better methodological quality.

Despite the limitations, our review has strengths and contributions to the literature. First, compared to the previous review that searched three databases [9], we searched six databases and the websites of four HTA agencies, with forward and backward citation searches. Second, our structured approach in examining the interventions and comparators revealed that the interventions and the comparators were often insufficiently described to provide clinical contexts being considered, and that most economic evaluations only accounted for consequences within the healthcare sector. These, in addition to the list of influential variables we presented would inform future economic evaluations on the topic.

\section{Conclusion}

Our review found ten studies, all model-based, of geneticguided pharmacotherapy for patients with VTE. With the heterogeneous interventions and comparators, gaps in quality of reporting and wide range in findings, it was not possible to pinpoint which type of genetic testing would be cost-effective or cost-saving. Several possible test strategies based on the guidelines and the literature were also notably missing. This includes testing based on family history and offering anticoagulation therapy in consideration of other risk factors in addition to test finding, testing genes recently discovered or testing both genes associated with higher risk of recurrent VTE and those associated with higher risk of bleeding. Besides examining these strategies and testing key model variables for decision uncertainty, future economic evaluations should improve the reporting of interventions, comparators and methodological details, to advise clinicians whether and when to adopt genetic-guided pharmacotherapy, especially with the discoveries of new high-risk genes and increasing availability of genetic testing.

Acknowledgements We thank Kamil Ahmad Amir for his assistance in updating the database searches and screening of titles, abstracts and full texts. We also thank King's College London librarians and Natalie Grant for help tracing full-text articles.

Author contributions RKK and JFR conceptualised and designed the study; RKK, CDAW, PC and JFR prepared the search strategies; RKK, JFR, and Kamil Ahmad Amir, a Master's student, undertook the searches and screening of abstracts and full texts. JFR, RKK and KKL prepared and piloted the data extraction tables, reviewed data extractions/quality assessments on two papers, and discussed any ratings that could not be agreed. RKK and KKL independently extracted the remaining data, rated the quality of included studies and prepared the first draft of the manuscript. KKL cleaned the data and performed the analyses. All authors contributed to data interpretation, revised the draft critically for important intellectual content and agreed to the final submission.

Funding The research was funded by the National Institute for Health Research (NIHR) Biomedical Research Centre based at Guy's and St Thomas' NHS Foundation Trust and King's College London. The views expressed are those of the authors and not necessarily those of the NHS, the NIHR or the Department of Health.

\section{Compliance with ethical standards}

Conflict of interest The authors declare no competing interests. 
Publisher's note Springer Nature remains neutral with regard to jurisdictional claims in published maps and institutional affiliations.

Open Access This article is licensed under a Creative Commons Attribution 4.0 International License, which permits use, sharing, adaptation, distribution and reproduction in any medium or format, as long as you give appropriate credit to the original author(s) and the source, provide a link to the Creative Commons license, and indicate if changes were made. The images or other third party material in this article are included in the article's Creative Commons license, unless indicated otherwise in a credit line to the material. If material is not included in the article's Creative Commons license and your intended use is not permitted by statutory regulation or exceeds the permitted use, you will need to obtain permission directly from the copyright holder. To view a copy of this license, visit http://creativecommons.org/licenses/by/4.0/.

\section{References}

1. Crous-Bou M, Harrington LB, Kabrhel C. Environmental and genetic risk factors associated with venous thromboembolism. Semin Thrombosis Hemost. 2016;42:808-20.

2. Stevens SM, Woller SC, Bauer KA, Kasthuri R, Cushman M, Streiff $\mathrm{M}$, et al. Guidance for the evaluation and treatment of hereditary and acquired thrombophilia. J Thrombosis Thrombolysis. 2016;41:154-64.

3. National Institute for Health and Care Excellence. Venous thromboembolic diseases: diagnosis, management and thrombophilia testing (NG158). UK; 2020.

4. Sanderson S, Emery J, Higgins J. CYP2C9 gene variants, drug dose, and bleeding risk in warfarin-treated patients: a HuGEnet systematic review and meta-analysis. Genet Med. 2005;7:97-104.

5. Zöller B, Svensson PJ, Dahlbäck B, Lind-Hallden C, Hallden C, Elf J. Genetic risk factors for venous thromboembolism. Expert Rev Hematol. 2020:13:971-81.

6. Lee E-J, Dykas DJ, Leavitt AD, Camire RM, Ebberink E, García de Frutos $\mathrm{P}$, et al. Whole-exome sequencing in evaluation of patients with venous thromboembolism. Blood Adv. 2017;1:1224-37.

7. Lindström S, Wang L, Smith EN, Gordon W, van Hylckama Vlieg A, de Andrade M, et al. Genomic and transcriptomic association studies identify 16 novel susceptibility loci for venous thromboembolism. Blood. 2019;134:1645-57.

8. Connors JM. Thrombophilia testing and venous thrombosis. N Engl J Med. 2017;377:1177-87.

9. Compagni A, Melegaro A, Tarricone R. Systematic review of the economic literature on genetic testing for the prevention of venous thromboembolism. Ital J Public Health. 2012;9:S36-246.

10. Fox-Rushby J, Koleva-Kolarova R, Chowienczyk P, Wolfe C. Systematic review of economic evaluations of stratified medicines for cardiovascular diseases: study protocol. 2019. https://www. crd.york.ac.uk/prospero/display_record.php?RecordID $=144579$.

11. Moher D, Liberati A, Tetzlaff J, Altman DG. Preferred reporting items for systematic reviews and meta-analyses: the PRISMA statement. BMJ. 2009;339:b2535. https://www.bmj.com/content/ 339/bmj.b2535.

12. Greenhalgh T, Peacock R. Effectiveness and efficiency of search methods in systematic reviews of complex evidence: audit of primary sources. BMJ. 2005;331:1064-5.

13. Hoffmann TC, Glasziou PP, Boutron I, Milne R, Perera R, Moher $\mathrm{D}$, et al. Better reporting of interventions: template for intervention description and replication (TIDieR) checklist and guide. Br Med J. 2014;348:g1687.

14. Neumann PJ, Ganiats TG, Russell LB, Sanders, GD, Siegel JE. Cost-effectiveness in health and medicine. 2nd ed. New York: Oxford University Press; 2016. p. 536.
15. Sanders GD, Neumann PJ, Basu A, Brock DW, Feeny D, Krahn $\mathrm{M}$, et al. Recommendations for conduct, methodological practices, and reporting of cost-effectiveness analyses: second panel on cost-effectiveness in health and medicine. JAMA. 2016;316:1093-103.

16. Evers SM, Goossens MEJB, Vet dHCW, Tulder vM, Ament A. Criteria list for assessment of methodological quality of economic evaluations:consensus on health economic criteria. Int $\mathbf{J}$ Technol Assess Health Care. 2005;21:240-5.

17. Odnoletkova I, Goderis G, Pil L, Nobels F, Aertgeerts B, Annemans $\mathrm{L}$, et al. Cost-effectiveness of therapeutic education to prevent the development and progression of type 2 diabetes: systematic review. J Diabetes Metab. 2014;5.

18. Nixon J, Khan KS, Kleijnen J. Summarising economic evaluations in systematic reviews: a new approach. BMJ. 2001;322: 1596-8.

19. Higashi MK, Veenstra DL. Managed care in the genomics era: assessing the cost effectiveness of genetic tests. Am J Managed Care. 2003;9:493-500.

20. Zhu Y, Swanson KM, Rojas RL, Wang Z, Sauver JLST, Visscher SL, et al. Systematic review of the evidence on the costeffectiveness of pharmacogenomics-guided treatment for cardiovascular diseases. Genet Med. 2020;22:475-86.

21. Eckman MH, Singh SK, Erban JK, Kao G. Testing for Factor V Leiden in patients with pulmonary or venous thromboembolism: a cost-effectiveness analysis. Med Decis Mak. 2002;22: $108-24$.

22. O'Brien SH, Smith KJ. Using thrombophilia testing to determine anticoagulation duration in pediatric thrombosis is not costeffective. J Pediatr. 2009;155:100-4.

23. You J, Tsui K, Wong R, Cheng G. Potential clinical and economic outcomes of CYP2C9 and VKORC1 genotype-guided dosing in patients starting warfarin therapy. Clin Pharmacol Therapeutics. 2009;86:540-7.

24. You JHS, Chan FWH, Wong RSM, Cheng G. The potential clinical and economic outcomes of pharmacogenetics-oriented management of warfarin therapy-a decision analysis. Thromb Haemost. 2004;92:590-7.

25. Auerbach AD, Sanders GD, Hambleton J. Cost-effectiveness of testing for hypercoagulability and effects on treatment strategies in patients with deep vein thrombosis. Am J Med. 2004;116:816-28.

26. Marchetti M, Pistorio A, Barosi G. Extended anticoagulation for prevention of recurrent venous thromboembolism in carriers of factor V Leiden-cost-effectiveness analysis. Thromb Haemost. 2000;84:752-7.

27. Marchetti M, Quaglini S, Barosi G. Cost-effectiveness of screening and extended anticoagulation for carriers of both factor $\mathrm{V}$ Leiden and prothrombin G20210A. QJM: Int J Med. 2001;94:365-72.

28. Schalekamp T, Boink GJJ, Visser LE, Stricker BHC, de Boer A, Klungel OH. CYP2C9 genotyping in acenocoumarol treatment: Is it a cost-effective addition to international normalized ratio monitoring?. Clin Pharmacol Therapeutics. 2006;79:511-20.

29. Rubio-Terrés C, Soria JM, Morange PE, Souto JC, Suchon P, Mateo $\mathrm{J}$, et al. Economic analysis of thrombo inCode, a clinical-genetic function for assessing the risk of venous thromboembolism. Appl Health Econ Health Policy. 2015;13:233-42.

30. Simpson EL, Stevenson MD, Rawdin A, Papaioannou D. Thrombophilia testing in people with venous thromboembolism: systematic review and cost-effectiveness analysis. Health Technol Assess. 2009;13:1-91.

31. Kimmel SE, French B, Kasner SE, Johnson JA, Anderson JL, Gage BF, et al. A pharmacogenetic versus a clinical algorithm for warfarin dosing. N Engl J Med. 2013;369:2283-93. 
32. Kheiri B, Abdalla A, Haykal T, Osman M, Ahmed S, Hassan M, et al. Meta-analysis of genotype-guided versus standard dosing of Vitamin K Antagonists. Am J Cardiol. 2018;121:879-87.

33. Belley-Cote EP, Hanif H, D’Aragon F, Eikelboom JW, Anderson JL, Borgman M, et al. Genotype-guided versus standard vitamin K antagonist dosing algorithms in patients initiating anticoagulation. A systematic review and meta-analysis. Thromb Haemost. 2015;114:768-77.

34. Cohn DM, Vansenne F, Kaptein AA, De Borgie CAJM, Middledorp $\mathrm{S}$, et al. The psychological impact of testing for thrombophilia: a systematic review. J Thrombosis Haemost. 2008;6:1099-104.
35. Lakdawalla DN, Doshi JA, Garrison LP Jr., Phelps CE, Basu A, Danzon PM. Defining elements of value in health care-a health economics approach: an ISPOR Special Task Force Report [3]. Value Health. 2018;21:131-9.

36. Lutsey PL, Walker RF, MacLehose RF, Alonso A, Adam TJ, Zakai NA. Direct oral anticoagulants and warfarin for venous thromboembolism treatment: trends from 2012 to 2017. Res Pr Thromb Haemost. 2019;3:668-73.

37. Loo SY, Dell'Aniello S, Huiart L, Renoux C. Trends in the prescription of novel oral anticoagulants in UK primary care. Br J Clin Pharmacol. 2017;83:2096-106. 